\title{
Usefulness of Adalimumab for Treating a Case of Intestinal Behçet's Disease With Trisomy 8 Myelodysplastic Syndrome
}

\author{
Masamichi Kimura ${ }^{1}$, Yoshihisa Tsuji ${ }^{1}$, Masako Iwai $^{1}$, Masahiro Inagaki ${ }^{1}$, Ali Madian ${ }^{2}$, Takuya Yoshino ${ }^{1}$, \\ Minoru Matsuura ${ }^{1}$, Hiroshi Nakase ${ }^{1}$ \\ Department of Gastroenterology and Hepatology, Kyoto University Hospital', Kyoto, Japan, Department of Internal Medicine, Al Azhar \\ University', Assint, Egypt
}

Behçet's disease (BD) is a systemic vasculitis, while myelodysplastic syndrome (MDS) is a heterogeneous group of clonal hematologic disorders characterized by ineffective hematopoiesis. Some studies suggest a relationship between MDS and BD, especially intestinal BD, and trisomy 8 seems to play an important role in both diseases. There are several reports on patients with BD comorbid with MDS involving trisomy 8 that frequently have intestinal lesions refractory to conventional medical therapies. Tumor necrosis factor (TNF)- $\alpha$ is strongly involved in the pathophysiology of several autoimmune diseases such as rheumatoid arthritis, inflammatory bowel disease, and BD. In addition, TNF- $\alpha$ plays an important role in the pathophysiology of MDS by inhibiting normal hematopoiesis and inducing the programmed cell death of normal total bone marrow cells and normal CD34+ cells. Recent clinical reports demonstrate the favorable effect of TNF- $\alpha$ antagonists in patients with refractory intestinal BD and in those with MDS. We present the case of a patient with intestinal BD and MDS involving trisomy 8 who was successfully treated with adalimumab. (Intest Res 2015;13:166-169)

Key Words: Adalimumab; Behçet syndrome; Trisomy 8; Myelodysplastic syndromes

\section{INTRODUCTION}

Behçet's disease (BD) is a chronic, relapsing, multisystem inflammatory disorder of unknown etiology, which is classified among the vasculitides. ${ }^{1}$ The cause of BD remains unknown; however, an autoimmune reaction triggered by an

Received December 15, 2014. Revised February 13, 2015.

Accepted February 27, 2015.

Correspondence to Yoshihisa Tsuji, Department of Gastroenterology and Hepatology, Kyoto University Hospital, 54 Kawahara-cho, Shogoin, Sakyoku, Kyoto 606-8507, Japan. Tel: +81-75-751-4302, Fax: +81-75-751-4303,

E-mail: ytsuji@kuhp.kyoto-u.ac.jp

Financial support: This work was supported by the Japanese Society for the Promotion of Science 8 involving (grant nos. 24590941, 25860532, 26460967, and 26893122) and also in part by the Health and Labor Sciences Research Grants for research on intractable diseases from the Ministry of Health, Labor, and Welfare of Japan (Investigation and Research for Intractable Inflammatory Bowel Disease). Conflict of interest: None. infectious agent in a genetically predisposed individual has been suggested. Recurrent oral ulcers in combination with genital ulcers, ocular disease, cutaneous lesions, arthritis, and less frequently, involvement of the gastrointestinal (GI) tract, central nervous system, and vascular beds have been typically observed. ${ }^{1}$ The disease can affect both sexes, and although it has a worldwide distribution, it is more prevalent in the Mediterranean and Far East.

Intestinal BD is characterized by intestinal ulcerations and GI symptoms. The incidence of BD involving the GI tract varies by country, ranging from 3-60\%. The GI lesions are often refractory to conventional medical therapies (e.g., corticosteroids [CS] and immunosuppressants), ${ }^{2}$ and they sometimes cause life-threatening comorbidities such as intestinal perforation and massive bleeding.

Myelodysplastic syndrome (MDS) is a heterogeneous

๑ Copyright 2015. Korean Association for the Study of Intestinal Diseases. All rights reserved.

This is an Open Access article distributed under the terms of the Creative Commons Attribution Non-Commercial License (http://creativecommons.org/licenses/by-nc/3.0)

which permits unrestricted non-commercial use, distribution, and reproduction in any medium, provided the original work is properly cited. 
group of clonal hematologic disorders characterized by ineffective hematopoiesis. Several reports suggest that patients with MDS and trisomy 8 are prone to having intestinal $\mathrm{BD}^{3-5} \mathrm{In}$ addition, intestinal BD patients with MDS involving trisomy 8 fail to respond to the aforementioned conventional medical therapies. Therefore, a new therapeutic strategy based on its pathogenesis is well expected for treating intestinal $\mathrm{BD}$ patients with MDS.

Tumor necrosis factor (TNF)- $\alpha$ is strongly involved in the pathophysiology of several autoimmune diseases such as rheumatoid arthritis, IBD, and BD. In addition, TNF- $\alpha$ plays an important role in the pathophysiology of MDS by inhibiting normal hematopoiesis and inducing the programmed cell death of normal total bone marrow cells and normal CD34+ cells. ${ }^{6}$ Recent clinical reports have demonstrated the favorable effect of TNF- $\alpha$ antagonists in patients with refractory intestinal BD and those with MDS. ${ }^{7}$

Considering the common pathophysiology of intestinal BD and MDS, the TNF- $\alpha$ antagonist may be an ideal drug for treating intestinal BD patients with MDS involving trisomy 8. However, there are no reports on treating intestinal BD and MDS successfully with TNF- $\alpha$ antagonist alone. Herein, we present the case of a patient with intestinal BD and MDS involving trisomy 8 who was successfully treated with adalimumab (ADA), a completely humanized IgG 1 monoclonal
anti-TNF- $\alpha$ antibody.

\section{CASE REPORT}

A 79-year-old female underwent endoscopic submucosal dissection (ESD) for an ileocecal, laterally spreading tumor. After ESD, she had a cecal refractory ulcer at the ESD site. Surgical resection of the ileocecal area was performed. However, the wound at the anastomotic site failed to heal, resulting in an enterocutaneous fistula. In addition, new ileal ulcers appeared at the anastomotic site. Moreover, the patient had intractable oral and genital ulcerations during her disease course. Despite no involvement of eye lesions or oral or genital ulcerations at this time, she had recurrent ulcers of the intestines with positive HLA-B51 alleles; thus, she was diagnosed with BD. Three months of prednisolone $(30 \mathrm{mg} /$ day) treatment without any other immune modulators relieved her upper abdominal pain. However, endoscopic findings revealed deterioration of the colonic ulcer (Fig. 1). She was admitted to our hospital for further treatment.

On admission, the physical examination revealed oral ulcers, genital ulcer scarring, and enterocutaneous fistulas. Blood tests showed that the white blood cell (WBC), hemoglobin $(\mathrm{Hb})$, and CRP levels were 6,500/ $\mu \mathrm{L}, 11.4 \mathrm{~g} / \mathrm{dL}$, and $0.6 \mathrm{mg} / \mathrm{dL}$, respectively. Firstly, surgical resection of the
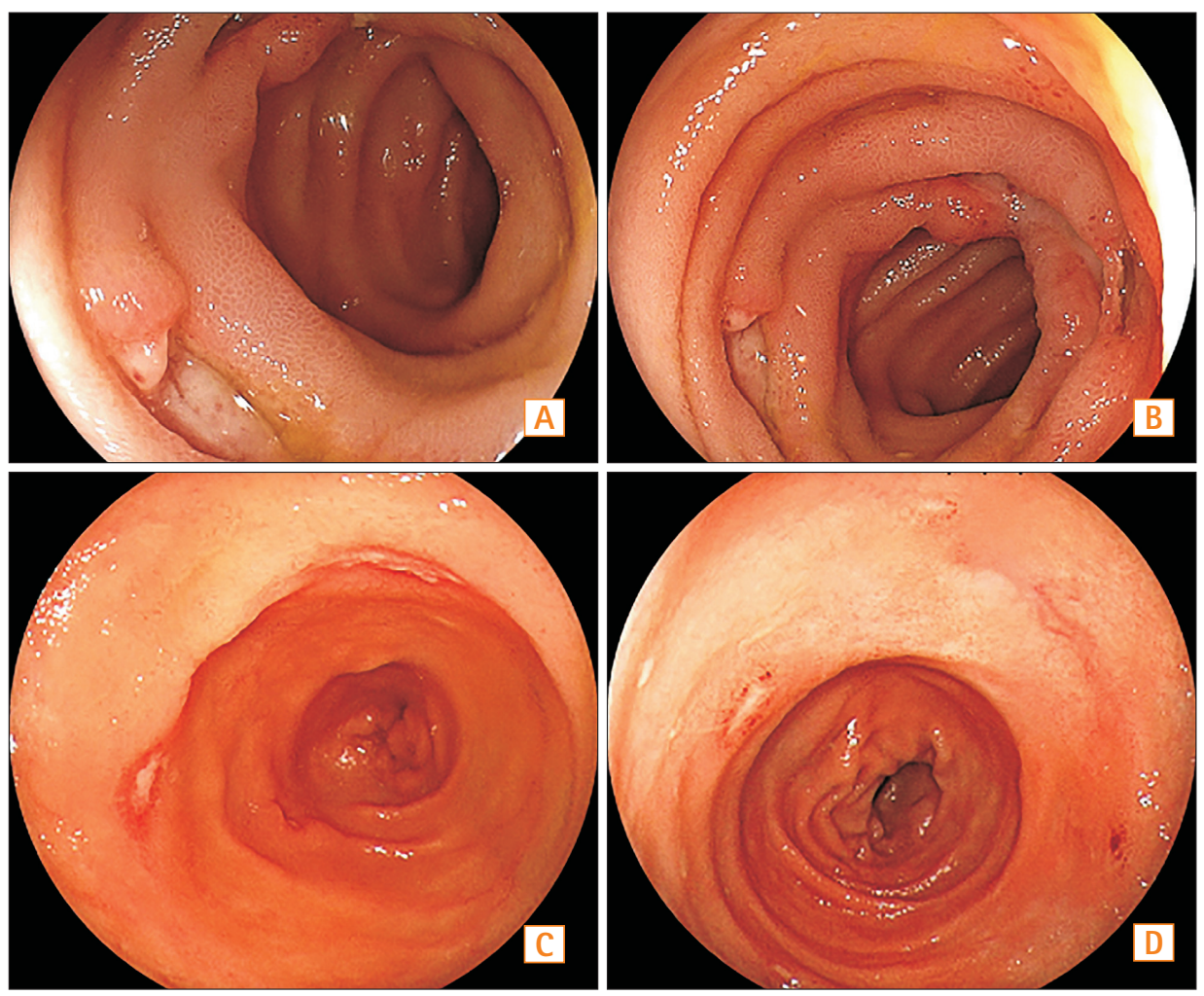

Fig. 1. Endoscopic findings before and after adalimumab (ADA) treatment. ( $\mathrm{A}$ and $\mathrm{B}$ ) Endoscopic findings prior to ADA treatment revealing redness, edematous mucosa, and multiple punched-out ulcers located at the postoperative anastomosis and the ileum. ( $C$ and D) Endoscopic findings showing marked improvement of the inflamed mucosa 1 year after starting ADA treatment. 
ileum and fistula closure was performed. However, her abdominal pain continued, and the laboratory data showed the following: leukocytopenia (WBC, 1,800/ $\mu \mathrm{L}$ ), anemia (Hb, 5.9 $\mathrm{g} / \mathrm{dL})$, and elevation of the CRP $(18.4 \mathrm{mg} / \mathrm{dL})$. She frequently required blood transfusion for severe anemia. A bone marrow biopsy was performed to further evaluate her continuous leukocytopenia and anemia. Bone marrow biopsy and aspiration revealed normocellular marrow with dysplasia. Conventional cytogenetic testing (G-banding) of her bone marrow demonstrated the presence of trisomy 8 in all the cells. These findings led to the diagnosis of MDS (refractory anemia with excess blasts-1) involving trisomy 8 . The international prognostic scoring system of BD was $4.75{ }^{8}$

Because of her diagnosis (i.e., CS-refractory BD associated with MDS involving trisomy 8), we administered ADA. We decided to only use adalimumab to treat MDS instead of using drugs such as azacitidine or decitabine, which may cause myelo-suppression, and she was an elderly patient with a high risk of infection. After the induction and maintenance therapy of ADA, her symptoms subsided (Fig. 1), and there was a striking improvement in her CRP level. Of note, a gradual improvement in her leukocytopenia and anemia was observed at 4 months after starting ADA. Presently, she does not require additional blood transfusions.

\section{DISCUSSION}

In this case, ADA was effective for treating intestinal BD and also anemia related to MDS with trisomy 8. To our knowledge, this is the first case of an intestinal BD patient with MDS involving trisomy 8 who was successfully treated with ADA. In addition, our case emphasizes that TNF- $\alpha$ plays a critical role in the pathogenesis of intestinal BD with MDS.

Until recently, there have been 39 reported cases of BD associated with MDS involving trisomy 8 . The trisomy 8 allele is one of the most frequent genetic abnormalities found in $6.5-16.3 \%$ of primary MDS patients. ${ }^{9}$ Furthermore, the prevalence rate of trisomy 8 is as high as $73.7 \%$ in MDS patients with $\mathrm{BD} .^{10}$ The majority of cases have incomplete types of BD (mainly the intestinal type) and the refractory anemia subtype of MDS. Generally, it has been reported that patients with intestinal BD with MDS involving trisomy 8 are refractory to conventional medical therapies.

Since extensive intramedullary cell death in MDS patients is related to TNF- $\alpha^{11,12}$ and the serum TNF- $\alpha$ concentrations are increased in BD patients, ${ }^{13}$ a TNF- $\alpha$ antagonist would theoretically be ideal for treating intestinal BD patients with
MDS. Currently, there are several reports on the effectiveness of TNF- $\alpha$ antagonists such as infliximab and ADA in patients with intestinal BD. A previous case of intestinal BD with MDS involving trisomy 8 was successfully treated with a combination of infliximab and methotrexate for an intestinal lesion; however, infliximab monotherapy was not effective for improving intestinal BD comorbid with MDS and trisomy $8^{14,15}$

When treating patients with BD who have severe symptoms related to deep ulcers of the intestine, CS are the firstline of induction therapy. However, in BD patients refractory to CS, the use of anti-TNF- $\alpha$ monoclonal antibodies would be considered, because there are many reports on the efficacy of anti-TNF- $\alpha$ monoclonal antibodies in managing intestinal BD; in addition, ADA was approved for intestinal BD in Japan in 2013. ${ }^{16}$ Moreover, many physicians avoid using a high dose of $\mathrm{CS}$ in elderly patients as much as possible. Therefore, in our case, we selected ADA as an alternative to increasing the dose of CS. We recommend anti-TNF- $\alpha$ therapy in patients with refractory $\mathrm{BD}$, although more data regarding the efficacy and safety of anti-TNF- $\alpha$ antibodies on refractory BD are needed.

It remains unclear whether infliximab is effective for intestinal BD comorbid with MDS involving trisomy 8. However, there are no previous reports on intestinal BD patients with MDS involving trisomy 8 who were treated with ADA. In our case, ADA monotherapy distinctly improved the patient's intestinal lesion and anemia. It is possible that the healing of the intestinal ulcers and good control of MDS with ADA may have contributed to the improvement of her anemia.

In conclusion, our case strongly suggests that intestinal BD patients with MDS involving trisomy 8 require optimal control of TNF- $\alpha$ using TNF- $\alpha$ antagonists. In the future, clinical trials with a larger number of patients will be required to elucidate whether ADA monotherapy is optimal for intestinal BD patients with MDS involving trisomy 8.

\section{REFERENCES}

1. Perra D, Alba MA, Callejas JL, et al. Adalimumab for the treatment of Behçet's disease: experience in 19 patients. Rheumatology (Oxford) 2012;51:1825-1831.

2. Ketch LL, Buerk CA, Liechty D. Surgical implications of Behçet's disease. Arch Surg 1980;115:759-760.

3. Ogawa $H$, Kuroda T, Inada M, et al. Intestinal Behçet's disease associated with myelodysplastic syndrome with chromosomal trisomy 8 -a report of two cases and a review of the literature. Hepatogastroenterology 2001;48:416-420. 
4. Kimura S, Kuroda J, Akaogi T, Hayashi H, Kobayashi Y, Kondo M. Trisomy 8 involved in myelodysplastic syndromes as a risk factor for intestinal ulcers and thrombosis-Behcet's syndrome. Leuk Lymphoma 2001;42:115-121.

5. Kawabata H, Sawaki T, Kawanami T, et al. Myelodysplastic syndrome complicated with inflammatory intestinal ulcers: significance of trisomy 8. Intern Med 2006;45:1309-1314.

6. Mundle SD, Ali A, Cartlidge JD, et al. Evidence for involvement of tumor necrosis factor- $\alpha$ in apoptotic death of bone marrow cells in myelodysplastic syndromes. Am J Hematol 1999;60:3647.

7. Naganuma M, Sakuraba A, Hisamatsu T, et al. Efficacy of infliximab for induction and maintenance of remission in intestinal Behçet's disease. Inflamm Bowel Dis 2008;14:1259-1264.

8. Greenberg P, Cox C, LeBeau MM, et al. International scoring system for evaluating prognosis in myelodysplastic syndromes. Blood 1997;89:2079-2088.

9. Chen G, Zeng W, Miyazato A, et al. Distinctive gene expression profiles of CD34 cells from patients with myelodysplastic syndrome characterized by specific chromosomal abnormalities. Blood 2004;104:4210-4218.

10. Lin YC, Liang TH, Chang HN, Lin JS, Lin HY. Behçet disease associated with myelodysplastic syndrome. J Clin Rheumatol 2008;14:169-174.
11. Campioni D, Secchiero P, Corallini F, et al. Evidence for a role of TNF-related apoptosis-inducing ligand (TRAIL) in the anemia of myelodysplastic syndromes. Am J Pathol 2005; 166:557-563.

12. Lee RW, Dick AD. Treat early and embrace the evidence in favour of anti-TNF- $\alpha$ therapy for Behçet's uveitis. Br J Ophthalmol 2010;94:269-270.

13. Duzgun N, Ayaslioglu E, Tutkak H, Aydintug OT. Cytokine inhibitors: soluble tumor necrosis factor receptor 1 and interleukin-1 receptor antagonist in Behçet's disease. Rheumatol Int 2005;25:1-5.

14. Toyonaga T, Nakase H, Matsuura M, et al. Refractoriness of intestinal Behçet's disease with myelodysplastic syndrome involving trisomy 8 to medical therapies - our case experience and review of the literature. Digestion 2013;88:217-221.

15. Iwata S, Saito K, Yamaoka K, et al. Efficacy of combination therapy of anti-TNF- $\alpha$ antibody infliximab and methotrexate in refractory entero-Behçet's disease. Mod Rheumatol 2011;21:184191.

16. Hisamatsu T, Ueno F, Matsumoto T, et al. The 2nd edition of consensus statements for the diagnosis and management of intestinal Behçet's disease: indication of anti-TNF $\alpha$ monoclonal antibodies. J Gastroenterol 2014;49:156-162. 